\title{
Regulation of traffic flows by railway stations with a change in the number of main tracks on the hauls
}

\author{
Aleksey Dmitrenko ${ }^{1, *}$, Arina Karaseva ${ }^{1}$, and Dmitriy Osipov ${ }^{1}$ \\ ${ }^{1}$ Siberian Transport University, Dusi Kovalchuk st., 191, 630049, Novosibirsk, Russia
}

\begin{abstract}
The theory of traffic flows suggests the possibility of using the analogy of handling transport units with hydraulic and other streaming processes. Railway stations, by analogy with HPPs, can be considered as compensation tanks, which ensure smoothing of fluctuations of the flows of transport units. The number of main tracks on the hauls affects the intensity and other parameters of traffic flows, which should be taken into account in calculating the track development of stations. The use of analogies with hydraulic flows allows proposing new methods for calculating the required capacity of compensating accumulators, i.e. track development of stations. In this regard, it is urgent to develop methods for correcting the calculation of the track development of stations in conditions of a change in the number of main tracks on the hauls. The purpose of the study was to develop a method for estimating the load at a station and the magnitude of the corresponding track development in conditions of a change in the number of main tracks on the hauls. The methods of simulation modeling and mathematical statistics are used. The result of the study will be a method for correcting the calculation of the track development of technical railway stations with a change in the number of main tracks on the hauls, as well as recommendations for estimating the stability of train traffic passing.
\end{abstract}

\section{Introduction}

In the historical aspect, with the advent of a new mode of transport - railway, it became possible to provide transportation of goods and passengers at a lower cost in compare with the previously existing horse and water transport modes. However, on railway transport, a need to develop methods for handling trains and find opportunities to develop increasing volumes of traffic appeared right in the beginning [1-9]. This required the development of railway lines - both hauls and interstations (stations). Traffic capacity of the station as a whole and its subsystems (leads, parks, etc.) is the most important characteristic of reliability of the transportation process $[10,16]$. In general, traffic capacity expresses the number of transport units (trains) that are passed (can be passed) through the station for a certain period of time.

\footnotetext{
* Corresponding author: dmitrenkoav@mail.ru
} 
The required traffic capacity ( $N_{r}$ is the number of transport units to be passed) is the analog of the "load" on the station as a system, and the available traffic capacity $\left(N_{a}\right.$ is the number of transport units that can be passed through with existing technical equipment, station operation technology and traffic management) - an analog of the "strength" of this system.

It is obvious that stable operation of the station is possible when $N_{a}>N_{r}$, otherwise the "load" exceeds "strength" and, naturally, there are failures in the work of the station as a system.

The concept of loading the station and its subsystems logically follows from the foregoing: the load is, in fact, the share of using the "strength" of the system, or the share (coefficient) of using the available traffic capacity.

At the initial stage of its development, new single-track trunk lines were mainly built. The presence of single-track railway lines instead of double-track lines made it possible to significantly reduce capital costs in the construction of new railways at the initial stage. Interstations, on which freight and technical operations (inspection of cars, change of locomotives) were carried out, were separated by single-track hauls. However, in the process of further operation of such lines, the need to develop increasing volumes of traffic was created. It became obvious that there was a close relationship between the operating conditions of the stations, the number of tracks at them, the development of the hauls, and the number of main tracks. Additional passing loops have been built to cross the trains. A number of authors have studied the development of rational constructions for passing loops, as well as for intermediate and precinct stations, the determination of the rational number of tracks at them, and also the design of parks $[9,13,16]$.

The development of increasing volumes of transportation for various stages of railway transport development was carried out in the following main directions:

1. By increasing the technical equipment of stations, increasing the number and length of station tracks, as well as construction of stations, taking into account the unevenness in traffic, especially in the case of performing overall repair of the track.

2. By increasing the traffic capacity of the hauls (primarily through the construction of the second main tracks) and increasing the traffic capacity of single-track railway lines.

3. By introduction of automatic interlocking on the hauls of the main railway lines.

4. By improving the design of stations for single-track railway lines.

5. For single-track railway lines, the passing loops have the task to increase the capacity. Thus, increasing the number of passing loops reduces the distance between them. This leads to a reduction in the period of the train traffic schedule and to an increase in the traffic and carriage capacity of the railway lines.

The nature of the passage of trains along the main single-track lines is influenced not only by the order of passing loops location. To a large extent, with the development of engineering, technology, and means of communication, the amount of costs was influenced by the used schemes of passing loops. Thus, the schemes of passing loops with transverse location of the receiving and departure tracks allow switching of points and preparation of routes for the receiving and departure of freight and passenger trains on single-track railway lines in a short period of time. However, with several parallel tracks on passing loop, in the case of the subsequent construction of continuous second tracks on previously single-track railway lines, additional tracks are unnecessary, which leads to an increase in the costs of reconstruction [13-15]. At the same time, passing loops with a transverse location scheme of the receiving and departure tracks cause increased operating costs associated with stopping freight trains when crossing them:

a) By increasing the time associated with the downtime of freight trains under the crossing, which is performed only with a stop. 
b) By energy consumption associated with braking and acceleration when stopping freight trains during their crossing.

c) By increasing the total number of crossings of freight trains.

When the size of traffic increases on single-track main lines, the costs associated with the crossing of freight trains on the passing loops significantly increase. Also, long delays of freight trains under crossing take place along the entire main railway line, if it all or a significant length of it is a single-track (for example, for the whole BAM: from Taishet to the Pacific Ocean). With the increase in traffic volumes, there appeared a need for delays of two trains from adjacent directions at once. In this regard, the need arose for a new order for the design of passing loops schemes with the longitudinal (sequential) location of the receiving and departure tracks on sites of great length. To reduce the total losses from freight train delays en route, increase the level of traffic and carriage capacity of the main lines, when developing the increasing volumes of traffic, it becomes expedient first of all to build a continuous second track on a single-track railway line. The increase in the number of main tracks on the hauls is traditionally regarded as a measure to increase the available traffic capacity. The solution of the problem of determining the rational track development of stations because of the use of a different number of main tracks on adjacent hauls can be proposed using a new approach based on the use of the analogy of passing traffic flows with flow in other systems, in particular, hydraulic ones (Figure 1). Movement of transport units is often compared with streaming processes $[1-4,17]$. In such systems, there are communications (channels) and storage tanks (for example, reservoirs of hydroelectric power stations $[5,8,11])$. On a smaller scale, traffic flow can be analyzed by analogy with piping type systems. The use of such an analogy makes it more understandable to determine the ways of development of existing calculation methods and the need to take into account various factors that affect the traffic capacity of the transport system, the development of hauls and stations.
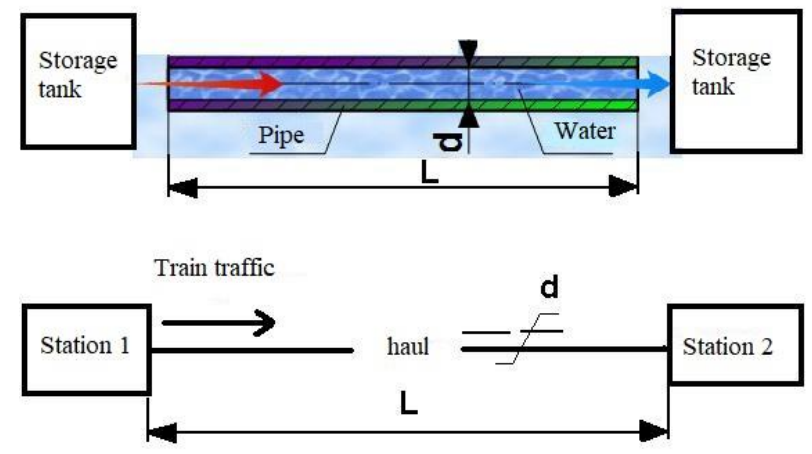

Fig. 1. The analogy of the transporting hydraulic system and the railway line element.

In accordance with the above, the purpose of the study is to develop a method for estimating the loads at the stations and the magnitude of the corresponding track development in conditions of a change in the number of main tracks in the presence of single-track hauls and single-track hauls with double-track inserted sections that perform the functions of passing loops for non-stop crossing of trains.

\section{Methods of research}

In the conditions of seasonal, intra-monthly, intra-day non-uniformity of freight train traffic, different duration of the same operations with specific trains, unevenness of train 
formation, influence of adjacent devices, failure of technical devices, and other objective factors, station tracks should ensure stable work of the station with high operational reliability $[6,12]$. The influence of these factors is estimated by the technically necessary proportion of the traffic capacity $\rho$ (for junction stations $\rho=0.4$ ), taking into account the size of the freight traffic along the station tracks, which ensure stable operation of the station. The coefficient of utilization of available track park capacity [6]:

$$
K_{\mathrm{p}}=\frac{T \cdot(1+\rho)}{\alpha \beta 1440 m-\sum T_{\text {perm }}^{\text {other }}},
$$

where $\alpha$ - coefficient that takes into account the influence on the use of the passenger (including suburban) and combined train tracks, $\beta=1$ for receiving and departure parks for transit trains;

$T$ - the total time of tracks' occupation by operations with freight trains, depending on the size of the traffic and the specialization of the tracks;

$\sum T_{\text {perm }}^{\text {other }}$ other permanent operations during the day that do not change in proportion to the size of traffic, and ongoing maintenance, planned types of repair and snow removal;

$\mathrm{m}$ - the number of receiving and departure tracks in the station parks (sections, groups).

The coefficient of utilization of the available capacity of the track park, based on its meaning, is an analog of the loading of the system (station), i.e. proportion of the use of available capacity. The available capacity of the park (section, group of tracks) to the accuracy of one transport unit:

$$
n_{i}^{n}=\frac{n_{i}}{K_{p}},
$$

where $n_{i}$ - the average daily number of routes of the i-th type.

It is important to note that the use of formula (1) does not allow us to identify the relationship between the capacity of the station tracks, their number and the number of main tracks on the hauls, while the analogy with the hydraulic system (Figure 1) makes it possible to determine such an effect quite simply.

With a significant difference in the characteristics of transport units (speed, load on the tracks, etc.), there are significant violations of the uniformity of the flow. Significant differences in the speed of the various participants in the flow lead to a loss of the total traffic capacity. In this case, the case of a "filled" flow, corresponding to a high degree of utilization of the traffic capacity of the railway line, is considered. They lead to a loss of the speed of its passage. An analogy for railway transport when passing mixed train traffic is the loss of capacity when passing trains with significantly different speeds and, correspondingly, hauling times. This leads to the appearance of so-called "removal" of trains of lower priority (and, as a rule, lower speed), proportional to the difference in train travel times and their number. Returning to the analogy with hydraulic transportation systems, this corresponds to the movement of a flow of different viscosities, which leads to the formation of turbulence that reduce the speed of its movement. When designing hydraulic systems, it is absolutely understandable that the design of transport systems for the passing of liquids of different viscosities, i.e. mixed, is unpractical. And it is difficult to imagine the freight corresponding to such a "mixture". In the operation of hydraulic systems, the flow is continuous, and a change in its volume corresponds to a decrease in pressure in the system. The traffic flow on the railway line is discrete (consists of separate trains), and a change in its volume corresponds to a reduction in the number of transport 
units. When considering the above analogy, the advantage of passing a uniform flow from the point of view of maximizing the traffic capacity of the transport system becomes evident. The uniform flow, with all other conditions being equal, moves faster than the mixed one. In hydraulic systems, the problem of ensuring the uniformity of the flow is solved by creating separate pipelines for liquids with different characteristics. On the railway line, the similar effect can be obtained by allocating separate main tracks for passing trains that significantly differ in terms of traffic conditions (primarily, speed characteristics) and the corresponding specialization of the main tracks of the multi-track lines. It is important to note that similar problems of mutual influence of various elements of the mixed flow occur with a high loading of the transport system (or high pressure in the hydraulic system). "Unfilled" systems (systems with low load, small use of available traffic capacity) will not have such problems. In this case, it is possible to allocate separate main tracks with their specialization for passing mixed transport units. An additional main track or tracks may appear with simultaneous specialization of the main tracks.

The diameter of the pipeline can be considered as an analog of the number of main tracks. The increase in the diameter is equivalent to the increase in the number of main tracks. An increase in the fluid flow rate corresponding to the increase in pressure reduces the need for the diameter of the pipeline. Considering the railway station as a reservoir creating a pressure in the transport system and compensating for its oscillation, it is possible to assume the opposite. Increased inlet pressure of the transport communication requires an increase in the speed of its movement. Conversely, an increase in the flow rate will create conditions for reducing the inlet pressure. "Pressure" here means the number of trains on the corresponding haul, i.e. the intensity of train preparation for departure. The laying of additional main tracks on the haul corresponds to an increase in the diameter of the pipeline, i.e. increase its traffic capacity, the pressure drop in it, the pressure drop at the inlet, reduction of the intensity of the station's work on the formation of the incoming flow for the adjacent transport communication, reduction of the required traffic and processing capacity of the station, its track development, reduction of the cost of track development and technical equipment of the station. A similar effect will have an increase in the speed of traffic flow through the transport communication and the possibility of using smaller capacity accumulators. Considering a railway station as a storage tank with an input and an output, it is possible to determine the conditions for minimizing its capacity. Constancy of the capacity is ensured when the outgoing and incoming flows are equal. Also, the required capacity decreases with an increase in the speed of the outflow. Increasing the diameter of the pipe leads to a drop in the level of liquid in the storage tanks due to the outlet of its part into the pipeline system. Taking into account the fact that the main task during the passage of the train traffic is to ensure the movement of the transported freight, the rationale will be such a scheme of transportations, in which the trains will go to the haul as soon as possible, i.e. will be in the phase of movement.

Obviously, an increase in the number of main tracks on the hauls adjacent to the station increases their traffic capacity and creates the conditions for a more rapid removal of trains from the station. Therefore, the laying of additional main tracks on the haul can be a resource for reducing the need for the track development of railway stations located on highly loaded lines. Further, the impact on the need for track development of the station of single-track hauls, as well as single-track hauls with double-track inserted sections used as passing loops for crossing trains of opposite directions, is considered. The required traffic capacity of the station and the corresponding number of receiving and departure tracks (see formula (1)) are proposed to be determined from the condition of equality of its traffic capacity - the traffic capacity of the adjacent line (single-track or single-track with doubletrack inserted sections). 
The need for single-track elements of the line is associated, first of all, with the solution of the problem of saving capital costs. When constructing a new railway line in order to save capital costs, the following design solutions are possible:

- design of single-track artificial structures (bridges, tunnels) instead of double-track ones;

- design of single-track hauls with double-track inserted sections.

Possible configurations for the line are shown in Figure 2 (A is a double-track line with the maximum level of traffic capacity and traffic safety, but with high capital investments; $\mathrm{B}$ is a single-track double-track version of the line, designing which it's possible to save, while ensuring a permissible level of traffic capacity and traffic safety).

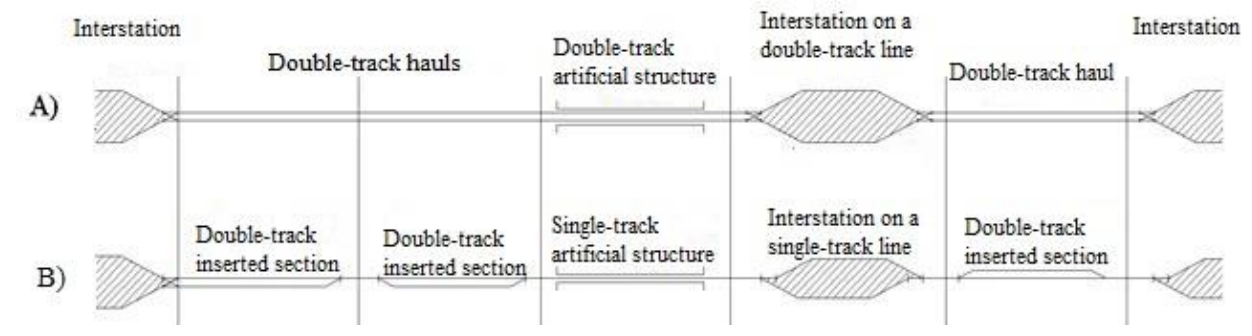

Fig. 2. Layout of the railway line using single-track and double-track elements.

Determination of configuration of the line, taking into account the different number of tracks on the hauls and railway stations, requires the use of both analytical calculations and the modeling of the train traffic passage on single-track hauls and hauls with double-track inserted sections.

\section{Results}

The traffic capacity of the single-track haul for freight trains Nfr passed through by groups depends on the number of trains in one group, $\mathrm{K}$, which is related to the number of block sections on the haul. An important factor affecting the traffic capacity of a single-track haul (or sequence of such hauls) is its length.

For the design conditions, with a length $\mathrm{L}$ of 6 to $18 \mathrm{~km}$ and the number of trains in the group $\mathrm{K}=2$, in this case [6], the traffic capacity is calculated by the formula:

$$
N_{a}=\frac{2\left(24-t_{\mathrm{tech}}\right) \alpha_{\mathrm{r}}}{\left[2-\alpha_{\mathrm{g}}\right]\left(t^{\prime}+t^{\prime \prime}+\tau_{A}+\tau_{B}\right)+\left(I^{\prime}+I^{\prime \prime}\right) \alpha_{\mathrm{g}}},
$$

where $t_{\text {tech }}$ - duration of the daily budget of time allocated for the implementation of planned repair and construction works, min.; $\alpha_{\mathrm{r}}-$ a coefficient that takes into account the reliability of operation of technical facilities; $K$ - the number of trains in the group, where $t^{\prime}, t^{\prime \prime}$ - the running time of odd and even trains in the single-track haul; $\tau_{\mathrm{A}}$ and $\tau_{B}$ are the station intervals for the interstations $\mathrm{A}$ and $\mathrm{B}$, provided by the train pass scheme; $I^{\prime}, I^{\prime \prime}-$ the calculated intervals between the trains passing in one direction in the group, respectively, in the odd and even directions; $\alpha_{\mathrm{g}}$ - the grouping coefficient $\alpha_{\mathrm{g}}=0,6$.

The level of traffic capacity is also influenced by the interval between the trains passing in one direction, which depends, with other conditions being equal, on the speed. The size of the intervals between the trains passing in one direction in the group depends on the speed of the train category under consideration, on the length of the train, and on the length of the block sections, and is 10 minutes for the speed of $60 \mathrm{~km} / \mathrm{h}$, for $80 \mathrm{~km} / \mathrm{h}-8$ minutes, respectively. 
Calculation of the traffic capacity of single-track hauls is carried out using the simulation model "Barrier-1" [7]. Graphical dependencies of the available traffic capacity on the length of a single-track element are shown in Figure 3.

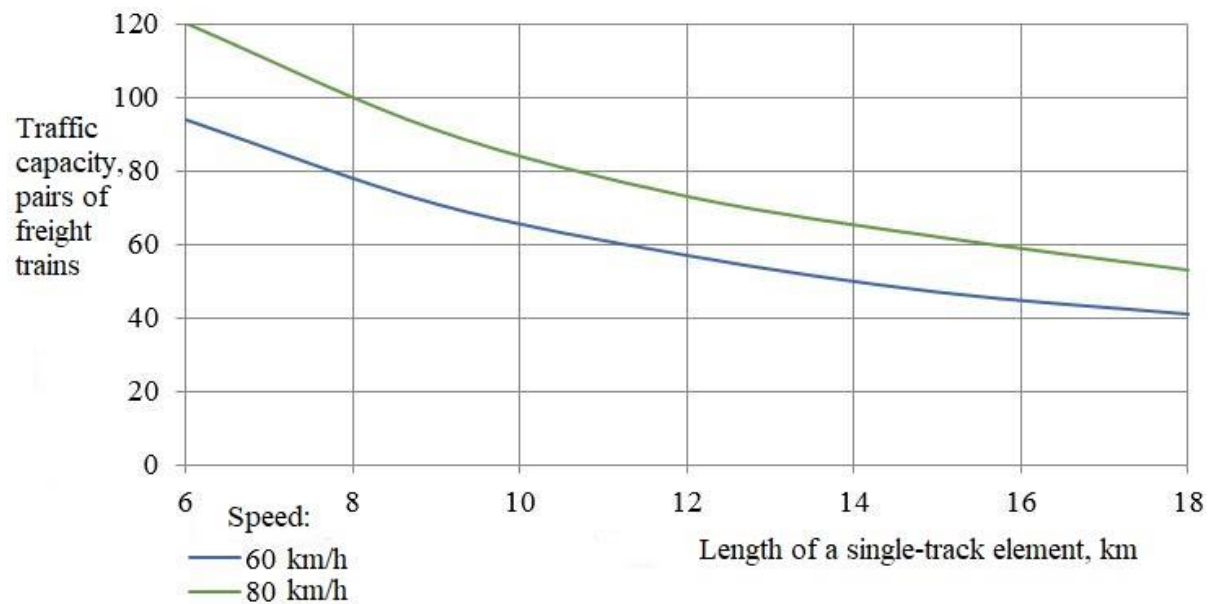

Fig. 3. Dependence of the maximum available traffic capacity on the length of a single track element of the railway line.

Using the obtained dependence curves of the traffic capacity on the length of a singletrack element, using regression analysis, the functional dependence of the form $f(x)=$ $a x^{3}+b x^{2}+c x+d$ was obtained. The third-order polynomial function most accurately describes the resulting graphical dependence.

The coefficients of the polynomial $(a, b, c, d)$ presented in Table 1 are determined for different speeds and duration of the period for passing trains of different categories.

Table 1. Coefficients of a polynomial for calculating the maximum available traffic capacity of a single-track line element and adjacent interstations.

\begin{tabular}{|c|c|c|c|c|}
\hline Average realizable speed of freight trains, $\mathrm{km} / \mathrm{h}$ & $\mathrm{a}$ & $\mathrm{b}$ & $\mathrm{c}$ & $\mathrm{d}$ \\
\hline 60 & $-0,0154$ & 0,8571 & $-17,766$ & 173 \\
\hline 80 & $-0,0278$ & 1,373 & $-25,536$ & 229,8 \\
\hline
\end{tabular}

The traffic capacity of a single-track haul with double-track inserted sections for freight trains can be determined by the formula:

$$
N_{\mathrm{a}}=\frac{\left(24-t_{\text {tech }}\right) \alpha_{\mathrm{r}}}{\mathrm{T}_{\text {haul }}}
$$

The values of the ultimate traffic capacity of a single-track haul with a double-track inserted section, which performs the function of passing loop for non-stop crossing, are shown in Figure 4. 


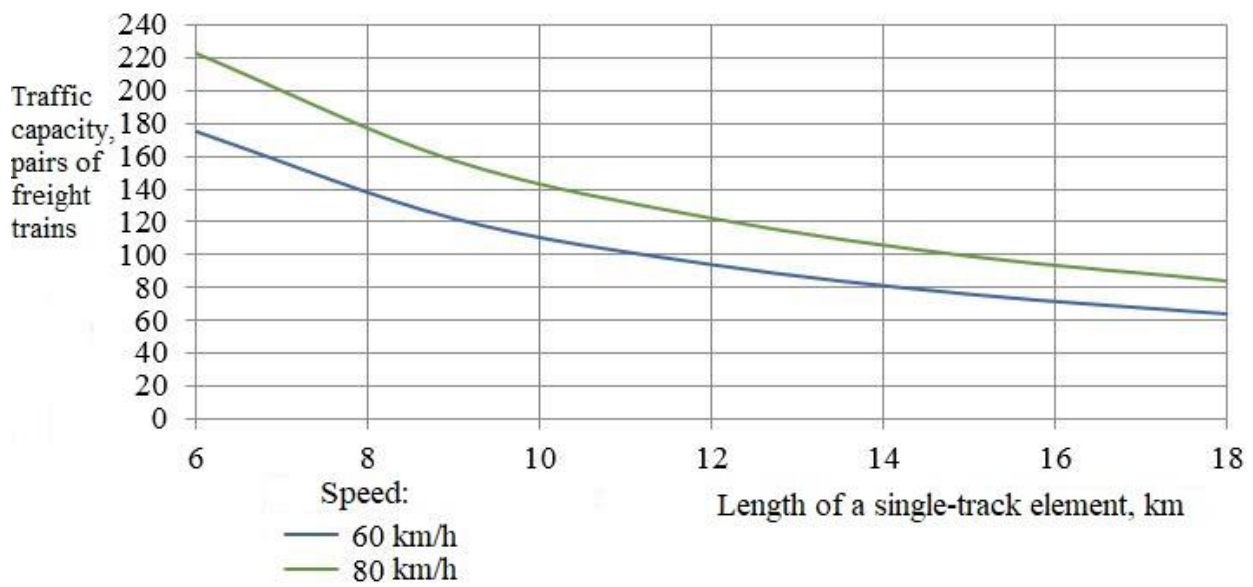

Fig. 4. Dependence of the maximum available traffic capacity from the length of a single-track railway line element in the presence of double-track inserted sections.

The functional dependence of the form $f(x)=a x^{3}+b x^{2}+c x+d$ is obtained. Coefficients of the polynomial function are presented in Table 2.

Table 2. Coefficients of a polynomial for calculating the ultimate available traffic capacity of a single-track line element with double-track inserted sections and adjacent interstations.

\begin{tabular}{|c|c|c|c|c|}
\hline $\begin{array}{c}\text { Average realizable speed of freight trains, } \\
\mathrm{km} / \mathrm{h}\end{array}$ & $\mathrm{a}$ & $\mathrm{b}$ & $\mathrm{c}$ & $\mathrm{d}$ \\
\hline 60 & $-0,0556$ & 2,7381 & $-49,048$ & 384,6 \\
\hline 80 & $-0,0741$ & 3,5714 & $-62,714$ & 488,6 \\
\hline
\end{tabular}

Analyzed dependencies of the traffic capacity of single-track hauls and single-track hauls with double-track inserted sections on the length and speed of trains passing by them can be used to justify pre-project solutions for line configuration in single-track two-track design, taking into account the required level of traffic capacity of both hauls and stations, carrying out the passage of train traffic, as well as with the feasibility study of the efficiency of high-speed traffic organization.

\section{Discussion}

The proposed approach to determining the need for track development of stations, on the basis of analogy with hydrodynamic systems, makes it possible to justify the need to take into account the conditions for the passage of train traffic along the railway lines, the number of main tracks on them. The optimal mode of operation of stations for regulating traffic flows is the mode that ensures the fastest letting out of trains to adjacent hauls. This mode should ensure the maximum proportion of trains on the move. In this case, the necessary track development of the stations will be minimal and, accordingly, the volume of capital investments in the development of expensive station infrastructure will decrease. The obtained results show that on single-track lines, the increase in traffic capacity of the lines can be achieved by increasing the overall level of speed, using shorter single-track hauls, and designing double-track inserted sections. Extended double-track inserted sections can perform the functions of passing loops and ensure the crossing of trains, including nonstop one. If it is necessary to increase traffic, the presence of double-track inserted sections will facilitate the change of the line into a double-track one. Calculations showed that an 
increase in the average speed by $30 \%$ provides an increase in the traffic capacity of singletrack elements of the route by $22-26 \%$. The use of double-track inserted sections creates a significant increase in the traffic capacity and an increase in the stability of the passage of train traffic along the railway lines. A good technological effect is given by the use of short single-track elements with double-track inserted sections. The greatest traffic capacity is ensured with a length of single-track hauls that does not exceed 6-8 km. Analytically obtained polynomial dependencies can be used to estimate the traffic capacity of the hauls and the required traffic capacity of the limiting stations. The method of correcting the calculation of the track development of railway stations with a change in the number of main tracks on the hauls will consist in ensuring that the number of receiving and departure tracks matches the capabilities of adjacent sections of the railway line in accordance with the results of calculating the traffic capacity of hauls of a single-track line, including double-track inserted sections. The latter can be designed separately or by extending the receiving and departure tracks of passing loops.

An important issue in this area, which requires further research, is the substantiation of the development features of large technical (sorting) stations with different traffic capacity for hauls of single- and multi-track lines, including in the conditions of the formation and departure of underweight and incomplete freight trains, as well as overall repairs of the track on the hauls. Another related area is the development of advance sections in large railway junctions using multi-track approaches.

\section{References}

1. S.G. Dagarizo, Fundamentals of transportation and traffic operations (Elsevier Science Inc., N.Y., 1997)

2. D.Helbing, Phys. Rev. E. 51, 3163-3169 (1995)

3. B.S. Kerner, Introduction to modern traffic flow theory and control. The long road to three-phase traffic theory (Springer, 2009)

4. P.M. Zhang, Transp. Res. B. 36, 275-290 (2002)

5. A.Yu. Aleksandrovskii, A.Yu. Soldatkin, Hydraulic engineering 8, 15-19 (2011)

6. Instructions for calculating the available traffic capacity of railways (Moscow, 2010)

7. A.D. Kalidova, Transport of the Urals 2(53), 102-108 (2010)

8. A.M. Kler, Z.R. Korneeva, P.Yu. Elsukov, Izvestiya of the Russian Academy of Sciences. Power engineering 2, 92-106 (2011)

9. D.Yu. Levin, Transportation capacities of railway transport. In the collection: Transport systems: development trends, 269-273 (2016)

10. D.Yu. Levin, System management of the transportation process in railway transport. Monograph (Scientific thought, Moscow, 2018)

11. I.V. Morozov, A.V. Gasnikov, V. Tarasov, Ya.A. Kholodov, A.S. Kholodov, Computer studies and modeling 3(4), 389-412 (2011)

12. Rules and technical standards for the design of stations and junctions on railways with gauge $1520 \mathrm{~mm}$ (Moscow, 2001)

13. B. Purev, A.V. Dmitrenko, Materials of the IX International Scientific and Technical Conference, 283-288 (2017)

14. B. Purev, A.V. Dmitrenko, In the collection: Transsib: on the edge of reforms. Materials of the international research and practice conference, 14-23 (2016) 
15. B. Purev, A.V. Dmitrenko, Transport infrastructure of the Siberian region 1, 12-16 (2017)

16. Yu.N. Fedorov, A.F. Borodin, Bulletin of the Joint Academic Council of JSC "Russian Railways" 4, 31-40 (2015)

17. Ya.A. Kholodov, A.S. Kholodov, A.V. Gasnikov, P.P. Morozov, V.I. Tarasov, Proceedings of MIPT 2-4(8), 152-162 (2010) 